\title{
Fibonacci Sayılarında Gizli Üçgensel Sayılar
}

Hidden Triangle Numbers in Fibonacci Numbers

Yiğit HESAPTAR ${ }^{1^{*}}$

Beste DENİ ${ }^{1}$

Nursen YILMAZ ${ }^{1}$

${ }^{1}$ Amasya Şehit Ferhat Ünelli Bilim ve Sanat Merkezi, Amasya / Türkiye

${ }^{1}$ Amasya Şehit Ferhat Ünelli Science and Art Center, Amasya / Turkey

*yigithesaptar@hotmail.com

ORCID: 0000-0001-5835-124X

ORCID: 0000-0002-3044-4388

ORCID: 0000-0001-6708-0656

\author{
MAKALE BILLGISİ / ARTICLE INFORMATION \\ Geliş Tarihi / Date Received \\ 15.10.2019 \\ Yayın Tarihi / Date Published \\ Temmuz / July 2020
}

\section{ATIF / CITE as}

Hesaptar, Y., Deniz, B. ve Yılmaz, N. (2020). “Fibonacci Sayılarında Gizli Üçgensel Sayılar" / "Hidden Triangle Numbers in Fibonacci Numbers". bilar: Bilim Armonisi Dergisi, 3 (1): 25-35. doi: 10.37215/ bilar.633484.

\section{https://dergipark.org.tr/tr/pub/bilar}

Copyright ( ) Published by Antalya İl Millî Eğitim Müdürlüğü Since 2018, Antalya, 07100 Turkey. All rights reserved. 


\section{ÖZET}

Bu araştırmada, “ $\{1,2,3,4,5, \ldots, n\}$ kümesinin herhangi iki ardışık tam sayı içermeyen kaç alt kümesi vardır?" probleminden yola çıkılarak Fibonacci Sayılarında gizli olan Üçgensel Sayılar keşfedilmiştir. "n. Fibonacci Sayısını oluşturan Üçgensel Sayıların düzeni nasıldır?" problemine cevap aranmıştır. \{1, 2, 3, $4,5, \ldots, \mathrm{n}\}$ kümesinin eleman sayısı sıfırdan başlatılarak $\mathrm{n}=12^{\prime}$ ye kadar incelenmiştir. Ardışık herhangi iki elemana sahip olmayan tüm alt kümeler sistematik olarak düzenlenerek çizelgeler oluşturulmuştur. n. Fibonacci Sayısını oluşturan Üçgensel Sayıların düzeni gösterilmiş ve Tümevarım Yöntemiyle ispat edilmiştir. Sonuç olarak Fibonacci Sayılarının Üçgensel Sayıların toplamı şeklinde yazılabildiği ve bu yazımda Üçgensel Sayıların katsayılarının da yine Fibonacci Sayılarından oluştuğu görülmüştür.

Anahtar Sözcükler: Fibonacci sayıları, Üçgensel sayılar, Alt küme.

\section{ABSTRACT}

In this research, "How many subsets of set $\{1,2,3,4,5, \ldots, \mathrm{n}\}$ have no two consecutive integers?" based on the problem, the Triangular Numbers hidden in the Fibonacci Numbers were discovered. The answer to the problem was sought "How should we write Triangular Numbers to compose n. Fibonacci?". The number of elements of: $\{1,2,3,4,5, \ldots, \mathrm{n}\}$ set is examined from $\mathrm{n}=0$ to $\mathrm{n}=12$. All subsets that do not have two consecutive elements are arranged systematically and charts are created. n. The order of the Triangular Numbers forming the Fibonacci Number is shown and proved by the Inductive Method. As a result, it has been observed that Fibonacci Numbers can be written as the sum of the Triangular Numbers, and in this article, the coefficients of the Triangular Numbers are also composed of Fibonacci Numbers.

Keywords: Fibonacci numbers, Triangular numbers, Subset. 


\section{Gikiş}

\subsection{Fibonacci Sayı Dizisi}

Fibonacci dizisi, her sayının kendinden hemen önceki iki sayının toplanması sonucu oluşan bir sayı dizisidir. Bu durumda, genel olarak n'inci Fibonacci Sayısı $\mathrm{F}_{\mathrm{n}}$ şu şekilde ifade edilir:

$$
\begin{aligned}
& \mathrm{F}_{1}=1, \\
& \mathrm{~F}_{2}=1, \\
& \mathrm{~F}_{\mathrm{n}}=\mathrm{F}_{\mathrm{n}-1}+\mathrm{F}_{\mathrm{n}-2} \quad \mathrm{n} \geq 3
\end{aligned}
$$

Buna göre Fibonacci Sayılarının ilk birkaç tanesi şöyle sıralanacaktır: $1,1,2,3,5,8,13,21,34,55$, $89,144,233,377,610,987,1597,2584,4181, \ldots$ (Sertöz 1997).

\section{2. Üçgensel Sayılar}

Bir Üçgensel Sayı, 1'den n'ye kadar olan n doğal sayının toplamıdır. Bu sayılara üçgensel denmesinin sebebi, bir üçgen şeklinde dizilebilecek eşit çaplı topların sayılarına karşılık gelmeleridir. n. Üçgensel Sayının formülü şöyledir:

$$
\ddot{\mathrm{U}}_{\mathrm{n}}=\sum_{\mathrm{k}=1}^{\mathrm{n}} \mathrm{k}=1+2+3+\ldots+(\mathrm{n}-1)+\mathrm{n}=\frac{\mathrm{n}(\mathrm{n}+1)}{2}
$$

İlk 20 Üçgensel Sayı şunlardır: 1, 3, 6, 10, 15, 21 , $28,36,45,55,66,78,91,105,120,136,153,171$ 190 ve 210 (Wells 1997).

$\mathrm{Bu}$ araştırmada, " $\{1,2,3,4,5, \ldots, \mathrm{n}\}$ kümesinin herhangi iki ardışık tam sayı içermeyen kaç alt kümesi vardır?" probleminden yola çıkılarak Fibonacci Sayılarında gizli olan Üçgensel Sayılar keşfedilmiştir. Böylece Fibonacci Sayıları için yeni bir eşitlik oluşturulmuştur.

2006 yılından 2018 yılına kadar yapılmış olan tüm çalışmalar incelendiğinde Fibonacci, Üçgensel Sayılar ve alt küme üzerine yapılmış bazı çalışmalara ulaşılmıştır. Fibonacci Sayıları ile ilgili bir dizi çalışma yapılmıştır (Uzun ve Keleştemur 2012, Koşar 2013). Bunlardan 2009 yılında yapılmış olan "Fibonacci Ağacı" isimli bir çalışmada, herhangi bir kümenin ardışık elemanlarının birlikte bulunmadığı alt küme sayısı üzerine çalışılmış ve çözüm için Fibonacci Sayı Dizisine ulaşılmıştır. Buradan hareketle bazı formüller bulunmuş ve Fibonacci Sayıları kombinasyonlar toplamı şeklinde ifade edilmiştir (Özer ve Ünlü 2009). Bulut (2017)'un yaptığı başka bir çalışmada da benzer sonuçlar elde edilmiş: Pascal Üçgeninden faydalanılarak Fibonacci Dizisinin n. elemanını doğrudan bulabilen bir formül önerilmiştir. Alt küme sayılarıyla ilgili 2015 yılında yapılan "Alt Kümelerin Üretici Fonksiyonları" isimli bir çalışmada, ardışık elemanlar bulunduran bir kümenin elemanları arasındaki fark 2'den büyük, 3'ten büyük ve k'den büyük olan durumlar incelenmiştir. Buna bağlı olarak da en az iki ardışık elemanı arasındaki fark $k$ olan $r$ elemanlı alt kümelerinin sayısını üretici fonksiyonlar yardımı ile bulunmuştur. Sonuçta alt küme sayıları kombinasyonlardan yararlanarak hesaplanmıştır (Takkın ve Korkmaz 2015). Ayrica, alt küme elemanlarının toplamını Pascal Üçgeninden bulma üzerine başka bir çalışma yapılmıştır (Taşgın ve Taşgın 2016). 2017 yılında yapılmış olan bir çalışmada ise parabol serilerinden Üçgensel Sayılara ulaşılmıştır (Aksoy ve Sarı 2017). Tüm araştırmalarımız sonucunda, Fibonacci Sayılarının Üçgensel Sayılar cinsinden ifadesine ulaşılamamıştır.

Çalışmamızda ise $\{1,2,3,4,5, \ldots, \mathrm{n}\}$ şeklindeki bir kümenin herhangi iki ardışık sayı içermeyen alt küme sayıları incelenirken ardışık sayı toplamları fark edilmiş ve Fibonacci Sayıları, Üçgensel Sayılar cinsinden gösterilmiştir.

\subsection{Amaç}

$\mathrm{Bu}$ çalışmada, " $\{1,2,3,4,5, \ldots, \mathrm{n}\}$ kümesinin herhangi iki ardışık tam sayı içermeyen kaç alt kümesi vardır?" probleminden yola çıkılarak bir Fibonacci Sayısında gizli olan Üçgensel Sayıları keşfetmek amaçlanmıştır. Alt problemler ise:

1. $\{1,2,3,4,5, \ldots, \mathrm{n}\}$ kümesinin herhangi iki ardışık tam sayı içermeyen kaç alt kümesi vardir?

2. n. Fibonacci Sayısını oluşturan Üçgensel Sayıların düzeni nasıldır? şeklindedir.

\section{MATERYAL VE METOT}

$\mathrm{A}=\{1,2,3,4,5, \ldots, \mathrm{n}\} \quad$ kümesinin bir alt kümesinin eleman sayısı sıfırdan başlatılarak $\mathrm{n}=12^{\prime}$ ye kadar incelenmiştir. Ardışık herhangi iki elemana sahip olmayan tüm alt kümeler sistematik olarak yazılarak, alt küme sayıları çizelgeler halinde düzenlenmiştir.

Özer ve Ünlü (2009) ile Bulut'un (2017) araştırmalarındaki gibi ardışık herhangi iki eleman bulundurmayan toplam alt küme sayısının bir Fibonacci Sayısı olduğu görülmüştür. Çizelgeler oluşturulurken çözümü oluşturan alt kümeler eleman sayılarına göre düzenlenmiştir. Yani sıfır elemanlı alt kümeler, 1 elemanlı, 2 elemanl1, ...vb. alt kümeler ayrı ayrı sütunlara yerleştirilmiştir. $\mathrm{Bu}$ sütunlar kendi içlerinde oluşturulurken de; içinde sayı değeri olarak en küçük “1" elemanını bulunduran, "2" elemanını bulunduran şeklinde satırlar halinde düzenlenince Üçgensel Sayıların varlığı fark edilmiştir. Öyleyse toplam sayı olan Fibonacci Sayısı, Üçgensel Sayılar cinsinden 
yazılabilmelidir. Herhangi ardışık iki eleman bulundurmayan toplam alt küme sayısını oluşturan Üçgensel Sayıların adetleri hesaplanarak ilgili Fibonacci Sayısına eşitliği gösterilmiştir. n. Fibonacci Sayısını oluşturan Üçgensel Sayıların düzeni gösterilmiş ve Tümevarım Yöntemiyle ispat edilmiştir.

\section{BULGULAR}

Sıfır elemanlı bir $A=\{\}$ kümesi için ardışık eleman içermeyen alt kümeler ve alt küme sayıları Çizelge 1'de gösterilmiştir.

Sıfır elemanlı bir kümenin alt kümesi de boş kümedir, alt küme sayısı da her daim 1 olacaktır. Bundan sonraki incelemede alt küme olarak boş kümeyi ifade eden 1 sayısı toplam alt küme sayısına eklenecektir.

Bir elemanlı bir $A=\{1\}$ kümesi için $n=1$ olup Çizelge 2'de alt kümeler gösterilmiştir.

Bir elemanlı alt küme sayısı da her daim kümenin eleman sayısına eşit olacağından, daima n'dir. Çizelge 2'ye göre ardışık herhangi iki eleman içermeyen toplam alt küme sayısı: $2=1+1=1+n$ olmaktadir.

İki elemanlı bir $\mathrm{A}=\{1,2\}$ kümesi için $\mathrm{n}=2$ olup Çizelge 3'te alt kümeler gösterilmiştir.

Ardışık herhangi eleman içermeyen toplam alt küme sayısı: $3=1+2=1+\mathrm{n}^{\prime}$ dir.

İki elemanlı bir kümenin alt kümelerinden kendisi, ardışık iki eleman içerdiğinden dolayı çözüm çizelgesine alınmamıştır.

Bundan sonraki incelemede boş küme ve bir elemanlı alt küme sayıları toplamını ifade eden $1+$ n sayısı, toplam alt küme sayısına eklenecektir. Alt küme sayılarının Üçgensel Sayılar ile ilişkisi üç elemanlı bir kümenin alt kümelerinde ilk defa fark edilecektir.

Üç elemanlı bir $\mathrm{A}=\{1,2,3\}$ kümesi için $\mathrm{n}=3$ olup Çizelge $4^{\prime}$ de alt kümeler gösterilmiştir.

Ardışık herhangi eleman içermeyen toplam alt küme sayısı: $5=1+3+1=1+\mathrm{n}+\ddot{\mathrm{U}}_{1}{ }^{\prime}$ dir.

Dört elemanlı bir $\mathrm{A}=\{1,2,3,4\}$ kümesi için $\mathrm{n}=4$ olup Çizelge $5^{\prime}$ te alt kümeler gösterilmiştir.

Ardışık herhangi iki eleman içermeyen toplam alt küme sayısı: $8=1+4+3=1+\mathrm{n}+\ddot{\mathrm{U}}_{2}{ }^{\prime}$ dir.

İki elemanlı alt kümeleri incelediğimizde içinde sayı değeri olarak en küçük "1" elemanı olan 2 alt küme; içinde en küçük " 2 " elemanı olan 1 alt küme olduğu görülür. $2+1=3=\ddot{U}_{2}{ }^{\prime}$ dir. Bu ardışık düzen çizelgelerde devam etmektedir.

Beş elemanlı bir $\mathrm{A}=\{1,2,3,4,5\}$ kümesi için $\mathrm{n}=5$ olup Çizelge $6^{\prime} \mathrm{da}$ alt kümeler gösterilmiştir.

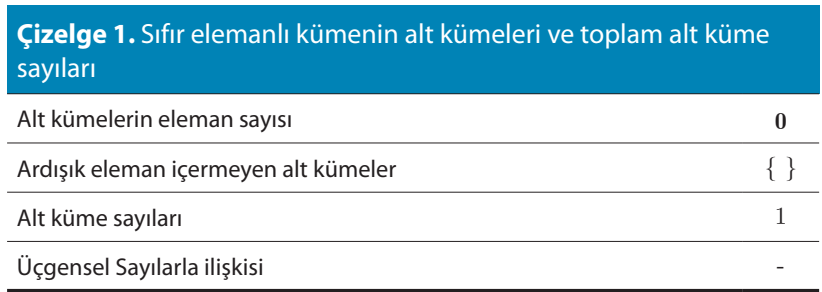

Çizelge 2. Bir elemanlı kümenin alt kümeleri ve toplam sayıları

\begin{tabular}{lcc} 
Alt kümelerin eleman sayısı & $\mathbf{0}$ & $\mathbf{1}$ \\
\hline Ardışık eleman içermeyen alt kümeler & \{\} & $\{1\}$ \\
\hline Alt küme sayılları & 1 & 1 \\
\hline Üçgensel Sayılarla ilişkisi & - & - \\
\hline
\end{tabular}

\section{Çizelge 3. Íki elemanlı kümenin alt kümeleri ve toplam sayıları}

\begin{tabular}{|c|c|c|c|}
\hline Alt kümelerin eleman sayısı & & 0 & 1 \\
\hline \multirow{2}{*}{ Ardışık eleman içermeyen alt kümeler } & \multirow{2}{*}{\multicolumn{2}{|c|}{}} & $\{1\}$ \\
\hline & & & $\{2\}$ \\
\hline Alt küme sayıları & & 1 & 2 \\
\hline Üçgensel Sayılarla ilişkisi & & - & - \\
\hline \multicolumn{4}{|c|}{$\begin{array}{l}\text { Çizelge 4. Üç elemanlı kümenin alt kümeleri, adetleri ve Üçgensel } \\
\text { Sayılarla ilişkisi }\end{array}$} \\
\hline Alt kümelerin eleman sayısı & 0 & 1 & 2 \\
\hline \multirow{3}{*}{ Ardışık eleman içermeyen alt kümeler } & \multirow[t]{3}{*}{} & $\{1\}$ & $\{1,3\}$ \\
\hline & & $\{2\}$ & \\
\hline & & $\{3\}$ & \\
\hline Alt küme sayıları & 1 & 3 & 1 \\
\hline Üçgensel Sayılarla ilişkisi & - & - & $\ddot{\mathrm{U}}_{1}$ \\
\hline
\end{tabular}

Çizelge 5. Dört elemanlı kümenin alt kümeleri, adetleri ve Üçgensel Sayılarla ilişkisi

\begin{tabular}{|c|c|c|c|c|c|}
\hline Alt kümelerin eleman sayısı & & & 0 & 1 & 2 \\
\hline & & & \{\} & $\{1\} \quad\{1$ & $\{1,3\},\{1,4\}$ \\
\hline Arduck oloman irormovon alt & & & & $\{2\} \quad\{2$ & $\{2,4\}$ \\
\hline 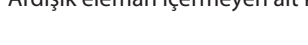 & & & & $\{3\}$ & \\
\hline & & & & $\{4\}$ & \\
\hline Alt küme sayıları & & & 1 & 4 & 3 \\
\hline Üçgensel Sayılarla ilişkisi & & & - & - & $\ddot{\mathrm{U}}_{2}$ \\
\hline $\begin{array}{l}\text { Çizelge 6. Beş elemanlı kü } \\
\text { Sayılarla ilişkisi }\end{array}$ & $\operatorname{nin}$ & küm & eleri, ad & tleri ve Ü & Üçgensel \\
\hline Alt kümelerin eleman sayısı & 0 & 1 & & 2 & 3 \\
\hline & \{\} & $\{1\}$ & $\{1,3\}$ & $1,4\},\{1,5\}$ & $5\} \quad\{1,3,5\}$ \\
\hline & & $\{2\}$ & $\{2,4\}$ & $2,5\}$ & \\
\hline $\begin{array}{l}\text { Ardısıık eleman içermeyen alt } \\
\text { kümeler }\end{array}$ & & $\{3\}$ & $\{3,5\}$ & & \\
\hline & & $\{4\}$ & & & \\
\hline & & $\{5\}$ & & & \\
\hline Alt küme sayıları & 1 & 5 & & 6 & 1 \\
\hline Üçgensel Sayılarla ilişkisi & - & - & & $\ddot{\mathrm{U}}_{3}$ & $\ddot{U}_{1}$ \\
\hline
\end{tabular}


Ardışık herhangi eleman içermeyen toplam alt küme sayısı: 13

$=1+5+6+1=1+\mathrm{n}+\ddot{\mathrm{U}}_{3}+\ddot{\mathrm{U}}_{1}^{\prime}$ dir.

İki elemanlı alt kümelerin $3+2+1$ şeklinde devam ettiği görülmektedir.

Altı elemanlı bir $\mathrm{A}=\{1,2,3,4,5,6\}$ kümesi için $\mathrm{n}=6$ olup Çizelge $7^{\prime}$ de alt kümeler gösterilmiştir.

\section{Çizelge 7. Altı elemanlı kümenin alt kümeleri, adetleri ve Üçgensel Sayılarla ilişkisi}

\begin{tabular}{|c|c|c|c|c|}
\hline Alt kümelerin eleman sayısı & 0 & 1 & 2 & 3 \\
\hline \multirow{6}{*}{ Ardışık eleman içermeyen alt kümeler } & \multirow[t]{6}{*}{} & $\{1\}$ & $\{1,3\},\{1,4\},\{1,5\},\{1,6\}$ & $\begin{array}{l}\{1,3,5\},\{1,3,6\} \\
\{1,4,6\}\end{array}$ \\
\hline & & $\{2\}$ & $\{2,4\},\{2,5\},\{2,6\}$ & $\{2,4,6\}$ \\
\hline & & $\{3\}$ & $\{3,5\},\{3,6\}$ & \\
\hline & & $\{4\}$ & $\{4,6\}$ & \\
\hline & & $\{5\}$ & & \\
\hline & & $\{6\}$ & & \\
\hline Alt küme sayıları & 1 & 6 & 10 & $3+1$ \\
\hline Üçgensel Sayılarla ilişkisi & - & - & $\ddot{\mathrm{U}}_{4}$ & $\ddot{\mathrm{U}}_{2}+\ddot{\mathrm{U}}_{1}$ \\
\hline
\end{tabular}

Ardışık herhangi eleman içermeyen toplam alt küme sayısı: 21

$=1+6+10+(3+1)=1+n+\ddot{U}_{4}+\left(\ddot{U}_{2}+\ddot{U}_{1}\right)^{\prime}$ dir.

İki elemanlı alt kümelerin $4+3+2+1$ şeklinde devam ederken; üç elemanlı alt kümelerden içinde 1 elemanını bulunduranlar, $\{1,3, \ldots\}$ ve $\{1,4, \ldots\}$ şeklinde ayrıldığında yine ardışık toplamlar $(2+1)$ elde edilmektedir.

Yedi elemanlı bir $\mathrm{A}=\{1,2,3,4,5,6,7\}$ kümesi için $\mathrm{n}=7$ olup Çizelge 8 ' de alt kümeler gösterilmiştir.

Çizelge 8. Yedi elemanlı kümenin alt kümeleri, adetleri ve Üçgensel Sayılarla ilişkisi

\begin{tabular}{|c|c|c|c|c|c|}
\hline Alt kümelerin eleman sayısı & 0 & 1 & 2 & 3 & 4 \\
\hline \multirow{7}{*}{ Ardışık eleman içermeyen alt kümeler } & \{\} & $\{1\}$ & $\{1,3\},\{1,4\},\{1,5\},\{1,6\},\{1,7\}$ & $\begin{array}{l}\{1,3,5\},\{1,3,6\},\{1,3,7\} \\
\{1,4,6\},\{1,4,7\} \\
\{1,5,7\}\end{array}$ & $\{1,3,5,7\}$ \\
\hline & & $\{2\}$ & $\{2,4\},\{2,5\},\{2,6\},\{2,7\}$ & $\begin{array}{l}\{2,4,6\},\{2,4,7\} \\
\{2,5,7\}\end{array}$ & \\
\hline & & $\{3\}$ & $\{3,5\},\{3,6\},\{3,7\}$ & $\{3,5,7\}$ & \\
\hline & & $\{4\}$ & $\{4,6\},\{4,7\}$ & & \\
\hline & & $\{5\}$ & $\{5,7\}$ & & \\
\hline & & $\{6\}$ & & & \\
\hline & & $\{7\}$ & & & \\
\hline Alt küme sayıları & 1 & 7 & 15 & $6+3+1$ & 1 \\
\hline Üçgensel Sayılarla ilişkisi & - & - & $\ddot{U}_{5}$ & $\ddot{U}_{3}+\ddot{U}_{2}+\ddot{U}_{1}$ & $\ddot{U}_{1}$ \\
\hline
\end{tabular}

Ardışık herhangi eleman içermeyen toplam alt küme sayısı: 34

$=1+7+15+(6+3+1)+1=1+\mathrm{n}+\ddot{\mathrm{U}}_{5}+\left(\ddot{\mathrm{U}}_{3}+\ddot{\mathrm{U}}_{2}+\ddot{\mathrm{U}}_{1}\right)+\ddot{\mathrm{U}}_{1}^{\prime}$ dir.

İki elemanlı alt kümeler $5+4+3+2+1=15=\ddot{U}_{5}$ şeklinde olup;

Üç elemanlı alt kümeler $(3+2+1)+(2+1)+1=\ddot{U}_{3}+\ddot{U}_{2}+\ddot{U}_{1}$ şeklindedir. 
Sekiz elemanlı bir $\mathrm{A}=\{1,2,3,4,5,6,7,8\}$ kümesi için $\mathrm{n}=8$ olup Çizelge 9' da alt kümeler gösterilmiştir. Çizelge 9. Sekiz elemanlı kümenin alt kümeleri, adetleri ve Üçgensel Sayılarla ilişkisi

\begin{tabular}{|c|c|c|c|c|c|}
\hline Alt kümelerin eleman sayısı & 0 & 1 & 2 & 3 & 4 \\
\hline \multirow{8}{*}{ Ardışık eleman içermeyen alt kümeler } & \{\} & $\{1\}$ & $\{1,3\},\{1,4\},\{1,5\},\{1,6\},\{1,7\},\{1,8\}$ & $\begin{array}{l}\{1,3,5\},\{1,3,6\},\{1,3,7\},\{1,3,8\} \\
\{1,4,6\},\{1,4,7\},\{1,4,8\} \\
\{1,5,7\},\{1,5,8\} \\
\{1,6,8\}\end{array}$ & $\begin{array}{l}\{1,3,5,7\},\{1,3,5,8\},\{1,3,6,8\} \\
\{1,4,6,8\}\end{array}$ \\
\hline & & $\{2\}$ & $\{2,4\},\{2,5\},\{2,6\},\{2,7\},\{2,8\}$ & $\begin{array}{l}\{2,4,6\},\{2,4,7\},\{2,4,8\} \\
\{2,5,7\},\{2,5,8\} \\
\{2,6,8\}\end{array}$ & $\{2,4,6,8\}$ \\
\hline & & $\{3\}$ & $\{3,5\},\{3,6\},\{3,7\},\{3,8\}$ & $\begin{array}{l}\{3,5,7\},\{3,5,8\} \\
\{3,6,8\}\end{array}$ & \\
\hline & & $\{4\}$ & $\{4,6\},\{4,7\},\{4,8\}$ & $\{4,6,8\}$ & \\
\hline & & $\{5\}$ & $\{5,7\},\{5,8\}$ & & \\
\hline & & $\{6\}$ & $\{6,8\}$ & & \\
\hline & & $\{7\}$ & & & \\
\hline & & $\{8\}$ & & & \\
\hline Alt küme sayıları & 1 & 8 & 21 & $10+6+3+1$ & $(3+1)+1$ \\
\hline Üçgensel Sayılarla ilişkisi & - & - & $\ddot{\mathrm{U}}_{6}$ & $\ddot{U}_{4}+\ddot{U}_{3}+\ddot{U}_{2}+\ddot{U}_{1}$ & $\left(\ddot{\mathrm{U}}_{2}+\ddot{\mathrm{U}}_{1}\right)+\ddot{\mathrm{U}}_{1}$ \\
\hline
\end{tabular}

Ardışık herhangi eleman içermeyen toplam alt küme sayısı: 55

$=1+8+21+(10+6+3+1)+(3+1)+1$

$=1+\mathrm{n}+\ddot{\mathrm{U}}_{6}+\left(\ddot{\mathrm{U}}_{4}+\ddot{\mathrm{U}}_{3}+\ddot{\mathrm{U}}_{2}+\ddot{\mathrm{U}}_{1}\right)+\left[\left(\ddot{\mathrm{U}}_{2}+\ddot{\mathrm{U}}_{1}\right)+\ddot{\mathrm{U}}_{1}\right]^{\prime} \mathrm{dir}$.

Dokuz elemanlı bir $\mathrm{A}=\{1,2,3,4,5,6,7,8,9\}$ kümesi için $\mathrm{n}=9$ olup Çizelge 10'da alt kümeler gösterilmiştir.

\section{Çizelge 10. Dokuz elemanlı kümenin alt kümeleri, adetleri ve Üçgensel Sayılarla ilişkisi}

\begin{tabular}{|c|c|c|c|c|c|c|}
\hline Alt kümelerin eleman sayısı & $\mathbf{0}$ & 1 & 2 & 3 & 4 & 5 \\
\hline \multirow{9}{*}{$\begin{array}{l}\text { Ardışık eleman içermeyen } \\
\text { alt kümeler }\end{array}$} & \{\} & $\{1\}$ & $\begin{array}{l}\{1,3\},\{1,4\},\{1,5\}, \\
\{1,6\},\{1,7\},\{1,8\},\{1,9\}\end{array}$ & $\begin{array}{l}\{1,3,5\},\{1,3,6\},\{1,3,7\},\{1,3,8\},\{1,3,9\} \\
\{1,4,6\},\{1,4,7\},\{1,4,8\},\{1,4,9\} \\
\{1,5,7\},\{1,5,8\},\{1,5,9\} \\
\{1,6,8\},\{1,6,9\} \\
\{1,7,9\}\end{array}$ & $\begin{array}{l}\{1,3,5,7\},\{1,3,5,8\},\{1,3,5,9\} \\
\{1,3,6,8\},\{1,3,6,9\},\{1,3,7,9\} \\
\{1,4,6,8\},\{1,4,6,9\},\{1,4,7,9\} \\
\{1,5,7,9\}\end{array}$ & $\{1,3,5,7,9\}$ \\
\hline & & $\{2\}$ & $\begin{array}{l}\{2,4\},\{2,5\},\{2,6\} \\
\{2,7\},\{2,8\},\{2,9\}\end{array}$ & $\begin{array}{l}\{2,4,6\},\{2,4,7\},\{2,4,8\},\{2,4,9\} \\
\{2,5,7\},\{2,5,8\},\{2,5,9\} \\
\{2,6,8\},\{2,6,9\} \\
\{2,7,9\}\end{array}$ & $\begin{array}{l}\{2,4,6,8\},\{2,4,6,9\},\{2,4,7,9\} \\
\{2,5,7,9\}\end{array}$ & \\
\hline & & $\{3\}$ & $\begin{array}{l}\{3,5\},\{3,6\},\{3,7\} \\
\{3,8\},\{3,9\}\end{array}$ & $\begin{array}{l}\{3,5,7\},\{3,5,8\},\{3,5,9\} \\
\{3,6,8\},\{3,6,9\} \\
\{3,7,9\}\end{array}$ & $\{3,5,7,9\}$ & \\
\hline & & $\{4\}$ & $\{4,6\},\{4,7\},\{4,8\},\{4,9\}$ & $\begin{array}{l}\{4,6,8\},\{4,6,9\} \\
\{4,7,9\}\end{array}$ & & \\
\hline & & $\{5\}$ & $\{5,7\},\{5,8\},\{5,9\}$ & $\{5,7,9\}$ & & \\
\hline & & $\{6\}$ & $\{6,8\},\{6,9\}$ & & & \\
\hline & & $\{7\}$ & $\{7,9\}$ & & & \\
\hline & & $\{8\}$ & & & & \\
\hline & & $\{9\}$ & & & & \\
\hline Alt küme sayıları & 1 & 9 & 28 & $15+10+6+3+1$ & $(6+3+1)+(3+1)+1$ & 1 \\
\hline Üçgensel Sayılarla ilişkisi & - & - & $\ddot{\mathrm{U}}_{7}$ & $\ddot{U}_{5}+\ddot{U}_{4}+\ddot{U}_{3}+\ddot{U}_{2}+\ddot{U}_{1}$ & $\left(\ddot{U}_{3}+\ddot{U}_{2}+\ddot{U}_{1}\right)+\left(\ddot{U}_{2}+\ddot{U}_{1}\right)+\ddot{U}_{1}$ & $\ddot{\mathrm{U}}_{1}$ \\
\hline
\end{tabular}

Ardışık herhangi eleman içermeyen toplam alt küme sayısı: 89

$$
\begin{aligned}
& =1+9+28+(15+10+6+3+1)+[(6+3+1)+(3+1)+1]+1 \\
& =1+n+\ddot{\mathrm{U}}_{7}+\left(\ddot{\mathrm{U}}_{5}+\ddot{\mathrm{U}}_{4}+\ddot{\mathrm{U}}_{3}+\ddot{\mathrm{U}}_{2}+\ddot{\mathrm{U}}_{1}\right)+\left[\left(\ddot{\mathrm{U}}_{3}+\ddot{\mathrm{U}}_{2}+\ddot{\mathrm{U}}_{1}\right)+\left(\ddot{\mathrm{U}}_{2}+\ddot{\mathrm{U}}_{1}\right)+\ddot{\mathrm{U}}_{1}\right]+\ddot{\mathrm{U}}_{1}{ }^{\prime} \text { dir. }
\end{aligned}
$$


On elemanlı bir $\mathrm{A}=\{1,2,3,4,5,6,7,8,9,10\}$ kümesi için $\mathrm{n}=10$ olup Çizelge $11^{\prime}$ de alt kümeler gösterilmiştir.

Çizelge 11. On elemanı kümenin alt kümeleri, adetleri ve Üçgensel Sayılarla ilişkisi

\begin{tabular}{|c|c|c|c|c|c|c|}
\hline $\begin{array}{l}\text { Alt kümelerin } \\
\text { eleman sayısı }\end{array}$ & 0 & 1 & 2 & 3 & 4 & 5 \\
\hline \multirow{10}{*}{$\begin{array}{l}\text { Ardışık eleman } \\
\text { içermeyen alt } \\
\text { kümeler }\end{array}$} & \multirow[t]{10}{*}{} & $\{1\}$ & $\begin{array}{l}\{1,3\}, \\
\{1,4\}, \\
\{1,5\}, \\
\{1,6\}, \\
\{1,7\}, \\
\{1,8\}, \\
\{1,9\}, \\
\{1,10\}\end{array}$ & $\begin{array}{l}\{1,3,5\},\{1,3,6\},\{1,3,7\}, \\
\{1,3,8\},\{1,3,9\},\{1,3,10\} \\
\{1,4,6\},\{1,4,7\},\{1,4,8\}, \\
\{1,4,9\},\{1,4,10\} \\
\{1,5,7\},\{1,5,8\},\{1,5,9\}, \\
\{1,5,10\} \\
\{1,6,8\},\{1,6,9\},\{1,6,10\} \\
\{1,7,9\},\{1,7,10\} \\
\{1,8,10\}\end{array}$ & $\begin{array}{l}\{1,3,5,7\},\{1,3,5,8\},\{1,3,5,9\},\{1,3,5,10\},\{1,3,6,8\}, \\
\{1,3,6,9\},\{1,3,6,10\},\{1,3,7,9\},\{1,3,7,10\},\{1,3,8,10\} \\
\{1,4,6,8\},\{1,4,6,9\},\{1,4,6,10\},\{1,4,7,9\},\{1,4,7,10\}, \\
\{1,4,8,10\} \\
\{1,5,7,9\},\{1,5,7,10\},\{1,5,8,10\}\end{array}$ & $\{2,4,6,8,10\}$ \\
\hline & & $\{2\}$ & $\begin{array}{l}\{2,4\}, \\
\{2,5\}, \\
\{2,6\}, \\
\{2,7\}, \\
\{2,8\}, \\
\{2,9\}, \\
\{2,10\}\end{array}$ & $\begin{array}{l}\{2,4,6\},\{2,4,7\},\{2,4,8\} \\
\{2,4,9\},\{2,4,10\} \\
\{2,5,7\},\{2,5,8\},\{2,5,9\} \\
\{2,5,10\} \\
\{2,6,8\},\{2,6,9\},\{2,6,10\} \\
\{2,7,9\},\{2,7,10\} \\
\{2,8,10\}\end{array}$ & $\begin{array}{l}\{2,4,6,8\},\{2,4,6,9\},\{2,4,6,10\},\{2,4,7,9\},\{2,4,7,10\} \\
\{2,5,7,9\},\{2,5,7,10\},,\{2,5,8,10\}\end{array}$ & \\
\hline & & $\{3\}$ & $\begin{array}{l}\{3,5\} \\
\{3,6\} \\
\{3,7\} \\
\{3,8\} \\
\{3,9\} \\
\{3,10\}\end{array}$ & $\begin{array}{l}\{3,5,7\},\{3,5,8\},\{3,5,9\} \\
\{3,5,10\} \\
\{3,6,8\},\{3,6,9\},\{3,6,10\} \\
\{3,7,9\},\{3,7,10\} \\
\{3,8,10\}\end{array}$ & $\begin{array}{l}\{3,5,7,9\},\{3,5,7,10\},\{3,5,8,10\} \\
\{3,6,8,10\}\end{array}$ & \\
\hline & & $\{4\}$ & $\begin{array}{l}\{4,6\} \\
\{4,7\} \\
\{4,8\} \\
\{4,9\} \\
\{4,10\}\end{array}$ & $\begin{array}{l}\{4,6,8\},\{4,6,9\},\{4,6,10\} \\
\{4,7,9\},\{4,7,10\} \\
\{4,8,10\}\end{array}$ & $\{4,6,8,10\}$ & \\
\hline & & $\{5\}$ & $\begin{array}{l}\{5,7\} \\
\{5,8\} \\
\{5,9\} \\
\{5,10\}\end{array}$ & $\begin{array}{l}\{5,7,9\},\{5,7,10\} \\
\{5,8,10\}\end{array}$ & & \\
\hline & & $\{6\}$ & $\begin{array}{l}\{6,8\} \\
\{6,9\} \\
\{6,10\}\end{array}$ & $\{6,8,10\}$ & & \\
\hline & & $\{7\}$ & $\begin{array}{l}\{7,9\} \\
\{7,10\}\end{array}$ & & & \\
\hline & & $\{8\}$ & $\{8,10\}$ & & & \\
\hline & & $\{9\}$ & & & & \\
\hline & & $\{10\}$ & & & & \\
\hline Alt küme sayıları & 1 & 10 & 36 & $21+15+10+6+3+1$ & $(10+6+3+1)+(6+3+1)+(3+1)+1$ & $(3+1)+1+1$ \\
\hline $\begin{array}{l}\text { Üçgensel Sayılarla } \\
\text { ilişkisi }\end{array}$ & - & - & $\ddot{U}_{8}$ & $\ddot{U}_{6}+\ddot{U}_{5}+\ddot{U}_{4}+\ddot{U}_{3}+\ddot{U}_{2}+\ddot{U}_{1}$ & $\left(\ddot{\mathrm{U}}_{4}+\ddot{\mathrm{U}}_{3}+\ddot{\mathrm{U}}_{2}+\ddot{\mathrm{U}}_{1}\right)+\left(\ddot{\mathrm{U}}_{3}+\ddot{\mathrm{U}}_{2}+\ddot{\mathrm{U}}_{1}\right)+\left(\ddot{\mathrm{U}}_{2}+\ddot{\mathrm{U}}_{1}\right)+\ddot{\mathrm{U}}_{1}$ & {$\left[\left(\ddot{U}_{2}+\ddot{U}_{1}\right)+\ddot{U}_{1}\right]+\ddot{U}_{1}$} \\
\hline
\end{tabular}

Ardışık herhangi eleman içermeyen toplam alt küme sayısı: 144

$$
\begin{aligned}
= & 1+10+36+(21+15+10+6+3+1)+[(10+6+3+1)+(6+3+1)+(3+1)+1]+(3+1) \\
& +1+1 \\
= & 1+\mathrm{n}+\ddot{\mathrm{U}}_{8}+\left(\ddot{\mathrm{U}}_{6}+\ddot{\mathrm{U}}_{5}+\ddot{\mathrm{U}}_{4}+\ddot{\mathrm{U}}_{3}+\ddot{\mathrm{U}}_{2}+\ddot{\mathrm{U}}_{1}\right)+\left[\left(\ddot{\mathrm{U}}_{4}+\ddot{\mathrm{U}}_{3}+\ddot{\mathrm{U}}_{2}+\ddot{\mathrm{U}}_{1}\right)+\left(\ddot{\mathrm{U}}_{3}+\ddot{\mathrm{U}}_{2}+\ddot{\mathrm{U}}_{1}\right)+\left(\ddot{\mathrm{U}}_{2}+\ddot{\mathrm{U}}_{1}\right)+\ddot{\mathrm{U}}_{1}\right]+ \\
& {\left[\left(\ddot{\mathrm{U}}_{2}+\ddot{\mathrm{U}}_{1}\right)+\ddot{\mathrm{U}}_{1}\right]+\ddot{\mathrm{U}}_{1}^{\prime} \text { dir. } } \\
\mathrm{n}= & 11 \text { için } \mathrm{A}=\{1,2,3,4,5,6,7,8,9,10,11\} \text { ve } \mathrm{n}=12 \text { için } \mathrm{A}=\{1,2,3,4,5,6,7,8,9,10,11,12\}
\end{aligned}
$$

kümeleri için de benzer çizelgeler oluşturulmuştur. Sonuç olarak hesaplanan alt küme sayıları:

$\mathrm{n}=11$ için $\mathrm{A}=\{1,2,3,4,5,6,7,8,9,10,11\}$ kümesinin ard1şık herhangi eleman içermeyen toplam alt küme sayısı: 233 


$$
\begin{aligned}
= & 1+\mathrm{n}+\ddot{\mathrm{U}}_{9}+\left(\ddot{\mathrm{U}}_{7}+\ddot{\mathrm{U}}_{6}+\ddot{\mathrm{U}}_{5}+\ddot{\mathrm{U}}_{4}+\ddot{\mathrm{U}}_{3}+\ddot{\mathrm{U}}_{2}+\ddot{\mathrm{U}}_{1}\right)+\left[\left(\ddot{\mathrm{U}}_{5}+\ddot{\mathrm{U}}_{4}+\ddot{\mathrm{U}}_{3}+\ddot{\mathrm{U}}_{2}+\ddot{\mathrm{U}}_{1}\right)+\left(\ddot{\mathrm{U}}_{4}+\ddot{\mathrm{U}}_{3}+\ddot{\mathrm{U}}_{2}+\ddot{\mathrm{U}}_{1}\right)+\right. \\
& \left.\left(\ddot{\mathrm{U}}_{3}+\ddot{\mathrm{U}}_{2}+\ddot{\mathrm{U}}_{1}\right)+\left(\ddot{\mathrm{U}}_{2}+\ddot{\mathrm{U}}_{1}\right)+\ddot{\mathrm{U}}_{1}\right]+\left[\left[\left(\ddot{\mathrm{U}}_{3}+\ddot{\mathrm{U}}_{2}+\ddot{\mathrm{U}}_{1}\right)+\left(\ddot{\mathrm{U}}_{2}+\ddot{\mathrm{U}}_{1}\right)+\ddot{\mathrm{U}}_{1}\right]+\left[\left(\ddot{\mathrm{U}}_{2}+\ddot{\mathrm{U}}_{1}\right)+\ddot{\mathrm{U}}_{1}\right]+\ddot{\mathrm{U}}_{1}\right]+\ddot{\mathrm{U}}_{1}^{\prime} \text { dir. }
\end{aligned}
$$

$\mathrm{n}=12$ için $\mathrm{A}=\{1,2,3,4,5,6,7,8,9,10,11,12\}$ kümesinin ard1şık herhangi eleman içermeyen toplam alt küme sayısı: 377

$$
\begin{aligned}
= & 1+n+\ddot{U}_{10}+\left(\ddot{U}_{8}+\ddot{U}_{7}+\ddot{U}_{6}+\ddot{U}_{5}+\ddot{U}_{4}+\ddot{U}_{3}+\ddot{U}_{2}+\ddot{U}_{1}\right)+\left[\left(\ddot{U}_{6}+\ddot{U}_{5}+\ddot{U}_{4}+\ddot{U}_{3}+\ddot{U}_{2}+\ddot{U}_{1}\right)+\right. \\
& \left.\left(\ddot{U}_{5}+\ddot{U}_{4}+\ddot{U}_{3}+\ddot{U}_{2}+\ddot{U}_{1}\right)+\left(\ddot{U}_{4}+\ddot{U}_{3}+\ddot{U}_{2}+\ddot{U}_{1}\right)+\left(\ddot{U}_{3}+\ddot{U}_{2}+\ddot{U}_{1}\right)+\left(\ddot{U}_{2}+\ddot{U}_{1}\right)+\ddot{U}_{1}\right]+ \\
& {\left[\left[\left(\ddot{U}_{4}+\ddot{U}_{3}+\ddot{U}_{2}+\ddot{U}_{1}\right)+\left(\ddot{U}_{3}+\ddot{U}_{2}+\ddot{U}_{1}\right)+\left(\ddot{U}_{2}+\ddot{U}_{1}\right)+\ddot{U}_{1}\right]+\left[\left[\left(\ddot{U}_{2}+\ddot{U}_{1}\right)+\ddot{U}_{1}\right]+\ddot{U}_{1}\right]+\ddot{U}_{1}\right] \text { şeklindedir. } }
\end{aligned}
$$

\subsection{Toplam Alt Küme Sayısının İçinde Bulunan Üçgensel Sayıların Adetleri}

Elde edilen toplam alt küme sayılarındaki Üçgensel Sayıları toparlarsak:

$$
\begin{aligned}
& \mathrm{n}=0 \text { için } 1+\mathrm{n} \\
& \mathrm{n}=1 \text { için } 1+\mathrm{n} \\
& \mathrm{n}=2 \text { için } 1+\mathrm{n} \\
& \mathrm{n}=3 \text { için } 1+\mathrm{n}+\ddot{\mathrm{U}}_{1} \\
& \mathrm{n}=4 \text { için } 1+\mathrm{n}+\ddot{\mathrm{U}}_{2} \\
& \mathrm{n}=5 \text { için } 1+\mathrm{n}+\ddot{\mathrm{U}}_{3}+1 . \ddot{\mathrm{U}}_{1} \\
& \mathrm{n}=6 \text { için } 1+\mathrm{n}+\ddot{\mathrm{U}}_{4}+1 . \ddot{\mathrm{U}}_{2}+1 . \ddot{\mathrm{U}}_{1} \\
& \mathrm{n}=7 \text { için } 1+\mathrm{n}+\ddot{\mathrm{U}}_{5}+1 . \ddot{\mathrm{U}}_{3}+1 . \ddot{\mathrm{U}}_{2}+2 . \ddot{\mathrm{U}}_{1} \\
& \mathrm{n}=8 \text { için } 1+\mathrm{n}+\ddot{\mathrm{U}}_{6}+1 . \ddot{\mathrm{U}}_{4}+1 . \ddot{\mathrm{U}}_{3}+2 \cdot \ddot{\mathrm{U}}_{2}+3 \cdot \ddot{\mathrm{U}}_{1} \\
& \mathrm{n}=9 \text { için } 1+\mathrm{n}+\ddot{\mathrm{U}}_{7}+1 \cdot \ddot{\mathrm{U}}_{5}+1 \cdot \ddot{\mathrm{U}}_{4}+2 \cdot \ddot{\mathrm{U}}_{3}+3 \cdot \ddot{\mathrm{U}}_{2}+5 \cdot \ddot{\mathrm{U}}_{1} \\
& \mathrm{n}=10 \text { için } 1+\mathrm{n}+\ddot{\mathrm{U}}_{8}+1 \cdot \ddot{\mathrm{U}}_{6}+1 \cdot \ddot{\mathrm{U}}_{5}+2 \cdot \ddot{\mathrm{U}}_{4}+3 \cdot \ddot{\mathrm{U}}_{3}+5 \cdot \ddot{\mathrm{U}}_{2}+8 \cdot \ddot{\mathrm{U}}_{1} \\
& \mathrm{n}=11 \text { için } 1+\mathrm{n}+\ddot{\mathrm{U}}_{9}+1 \cdot \ddot{\mathrm{U}}_{7}+1 \cdot \ddot{\mathrm{U}}_{6}+2 \cdot \ddot{\mathrm{U}}_{5}+3 \cdot \ddot{\mathrm{U}}_{4}+5 \cdot \ddot{\mathrm{U}}_{3}+8 \cdot \ddot{\mathrm{U}}_{2}+13 \cdot \ddot{\mathrm{U}}_{1} \\
& \mathrm{n}=12 \text { için } 1+\mathrm{n}+\ddot{\mathrm{U}}_{10}+1 \cdot \ddot{\mathrm{U}}_{8}+1 \cdot \ddot{\mathrm{U}}_{7}+2 \cdot \ddot{\mathrm{U}}_{6}+3 \cdot \ddot{\mathrm{U}}_{5}+5 \cdot \ddot{\mathrm{U}}_{4}+8 \cdot \ddot{\mathrm{U}}_{3}+13 \cdot \ddot{\mathrm{U}}_{2}+21 \cdot \ddot{\mathrm{U}}_{1}
\end{aligned}
$$

\section{2. Üçgensel Sayıların Katsayılarının Fibonacci Sayısı Olarak ifade Edilmesi}

Üçgensel Sayılarının katsayılarının yine bir Fibonacci Sayısı olduğu görülmektedir. Öyleyse toplam alt küme sayılarını tekrar düzenlersek:

$$
\begin{aligned}
& \mathrm{n}=0 \text { için } 1+\mathrm{n} \\
& \mathrm{n}=1 \text { için } 1+\mathrm{n} \\
& \mathrm{n}=2 \text { için } 1+\mathrm{n} \\
& \mathrm{n}=3 \text { için } 1+\mathrm{n}+\ddot{\mathrm{U}}_{1} \\
& \mathrm{n}=4 \text { için } 1+\mathrm{n}+\ddot{\mathrm{U}}_{2} \\
& \mathrm{n}=5 \text { için } 1+\mathrm{n}+\ddot{\mathrm{U}}_{3}+\mathrm{F}_{1} \cdot \ddot{\mathrm{U}}_{1} \\
& \mathrm{n}=6 \text { için } 1+\mathrm{n}+\ddot{\mathrm{U}}_{4}+\mathrm{F}_{1} \cdot \ddot{\mathrm{U}}_{2}+\mathrm{F}_{2} \cdot \ddot{\mathrm{U}}_{1} \\
& \mathrm{n}=7 \text { için } 1+\mathrm{n}+\ddot{\mathrm{U}}_{5}+\mathrm{F}_{1} \cdot \ddot{\mathrm{U}}_{3}+\mathrm{F}_{2} \cdot \ddot{\mathrm{U}}_{2}+\mathrm{F}_{3} \cdot \ddot{\mathrm{U}}_{1} \\
& \mathrm{n}=8 \text { için } 1+\mathrm{n}+\ddot{\mathrm{U}}_{6}+\mathrm{F}_{1} \cdot \ddot{\mathrm{U}}_{4}+\mathrm{F}_{2} \cdot \ddot{\mathrm{U}}_{3}+\mathrm{F}_{3} \cdot \ddot{\mathrm{U}}_{2}+\mathrm{F}_{4} \cdot \ddot{\mathrm{U}}_{1} \\
& \mathrm{n}=9 \text { için } 1+\mathrm{n}+\ddot{\mathrm{U}}_{7}+\mathrm{F}_{1} \cdot \ddot{\mathrm{U}}_{5}+\mathrm{F}_{2} \cdot \ddot{\mathrm{U}}_{4}+\mathrm{F}_{3} \cdot \ddot{\mathrm{U}}_{3}+\mathrm{F}_{4} \cdot \ddot{\mathrm{U}}_{2}+\mathrm{F}_{5} \cdot \ddot{\mathrm{U}}_{1}
\end{aligned}
$$


$\mathrm{n}=10$ için $1+\mathrm{n}+\ddot{\mathrm{U}}_{8}+\mathrm{F}_{1} \cdot \ddot{\mathrm{U}}_{6}+\mathrm{F}_{2} \cdot \ddot{\mathrm{U}}_{5}+\mathrm{F}_{3} \cdot \ddot{\mathrm{U}}_{4}+\mathrm{F}_{4} \cdot \ddot{\mathrm{U}}_{3}+\mathrm{F}_{5} \cdot \ddot{\mathrm{U}}_{2}+\mathrm{F}_{6} \cdot \ddot{\mathrm{U}}_{1}$

$\mathrm{n}=11$ için $1+\mathrm{n}+\ddot{\mathrm{U}}_{9}+\mathrm{F}_{1} \cdot \ddot{\mathrm{U}}_{7}+\mathrm{F}_{2} \cdot \ddot{\mathrm{U}}_{6}+\mathrm{F}_{3} \cdot \ddot{\mathrm{U}}_{5}+\mathrm{F}_{4} \cdot \ddot{\mathrm{U}}_{4}+\mathrm{F}_{5} \cdot \ddot{\mathrm{U}}_{3}+\mathrm{F}_{6} \cdot \ddot{\mathrm{U}}_{2}+\mathrm{F}_{7} \cdot \ddot{\mathrm{U}}_{1}$

$\mathrm{n}=12$ için $1+\mathrm{n}+\ddot{\mathrm{U}}_{10}+\mathrm{F}_{1} \cdot \ddot{\mathrm{U}}_{8}+\mathrm{F}_{2} \cdot \ddot{\mathrm{U}}_{7}+\mathrm{F}_{3} \cdot \ddot{\mathrm{U}}_{6}+\mathrm{F}_{4} \cdot \ddot{\mathrm{U}}_{5}+\mathrm{F}_{5} \cdot \ddot{\mathrm{U}}_{4}+\mathrm{F}_{6} \cdot \ddot{\mathrm{U}}_{3}+\mathrm{F}_{7} \cdot \ddot{\mathrm{U}}_{2}+\mathrm{F}_{8} \cdot \ddot{\mathrm{U}}_{1}$

düzenini elde ederiz.

\subsection{Bir Fibonacci Sayısının Üçgensel Sayılar Cinsinden Ifadesi}

Herhangi iki ardışık eleman içermeyen toplam alt küme sayısının bir Fibonacci Sayısı olduğu (Özer ve Ünlü 2009) bilindiğine göre:

$$
\begin{aligned}
& \mathrm{n}=0 \text { için } 1+\mathrm{n}=\mathrm{F}_{2} \\
& \mathrm{n}=1 \text { için } 1+\mathrm{n}=\mathrm{F}_{3} \\
& \mathrm{n}=2 \text { için } 1+\mathrm{n}=\mathrm{F}_{4} \\
& \mathrm{n}=3 \text { için } 1+\mathrm{n}+\ddot{\mathrm{U}}_{1}=\mathrm{F}_{5} \\
& \mathrm{n}=4 \text { için } 1+\mathrm{n}+\ddot{\mathrm{U}}_{2}=\mathrm{F}_{6} \\
& \mathrm{n}=5 \text { için } 1+\mathrm{n}+\ddot{\mathrm{U}}_{3}+\mathrm{F}_{1} \cdot \ddot{\mathrm{U}}_{1}=\mathrm{F}_{7} \\
& \mathrm{n}=6 \text { için } 1+\mathrm{n}+\ddot{\mathrm{U}}_{4}+\mathrm{F}_{1} \cdot \ddot{\mathrm{U}}_{2}+\mathrm{F}_{2} \cdot \ddot{\mathrm{U}}_{1}=\mathrm{F}_{8} \\
& \mathrm{n}=7 \text { için } 1+\mathrm{n}+\ddot{\mathrm{U}}_{5}+\mathrm{F}_{1} \cdot \ddot{\mathrm{U}}_{3}+\mathrm{F}_{2} \cdot \ddot{\mathrm{U}}_{2}+\mathrm{F}_{3} \cdot \ddot{\mathrm{U}}_{1}=\mathrm{F}_{9} \\
& \mathrm{n}=8 \text { için } 1+\mathrm{n}+\ddot{\mathrm{U}}_{6}+\mathrm{F}_{1} \cdot \ddot{\mathrm{U}}_{4}+\mathrm{F}_{2} \cdot \ddot{\mathrm{U}}_{3}+\mathrm{F}_{3} \cdot \ddot{\mathrm{U}}_{2}+\mathrm{F}_{4} \cdot \ddot{\mathrm{U}}_{1}=\mathrm{F}_{10} \\
& \mathrm{n}=9 \text { için } 1+\mathrm{n}+\ddot{\mathrm{U}}_{7}+\mathrm{F}_{1} \cdot \ddot{\mathrm{U}}_{5}+\mathrm{F}_{2} \cdot \ddot{\mathrm{U}}_{4}+\mathrm{F}_{3} \cdot \ddot{\mathrm{U}}_{3}+\mathrm{F}_{4} \cdot \ddot{\mathrm{U}}_{2}+\mathrm{F}_{5} \cdot \ddot{\mathrm{U}}_{1}=\mathrm{F}_{11} \\
& \mathrm{n}=10 \text { için } 1+\mathrm{n}+\ddot{\mathrm{U}}_{8}+\mathrm{F}_{1} \cdot \ddot{\mathrm{U}}_{6}+\mathrm{F}_{2} \cdot \ddot{\mathrm{U}}_{5}+\mathrm{F}_{3} \cdot \ddot{\mathrm{U}}_{4}+\mathrm{F}_{4} \cdot \ddot{\mathrm{U}}_{3}+\mathrm{F}_{5} \cdot \ddot{\mathrm{U}}_{2}+\mathrm{F}_{6} \cdot \ddot{\mathrm{U}}_{1}=\mathrm{F}_{12} \\
& \mathrm{n}=11 \text { için } 1+\mathrm{n}+\ddot{\mathrm{U}}_{9}+\mathrm{F}_{1} \cdot \ddot{\mathrm{U}}_{7}+\mathrm{F}_{2} \cdot \ddot{\mathrm{U}}_{6}+\mathrm{F}_{3} \cdot \ddot{\mathrm{U}}_{5}+\mathrm{F}_{4} \cdot \ddot{\mathrm{U}}_{4}+\mathrm{F}_{5} \cdot \ddot{\mathrm{U}}_{3}+\mathrm{F}_{6} \cdot \ddot{\mathrm{U}}_{2}+\mathrm{F}_{7} \cdot \ddot{\mathrm{U}}_{1}=\mathrm{F}_{13} \\
& \mathrm{n}=12 \text { için } 1+\mathrm{n}+\ddot{\mathrm{U}}_{10}+\mathrm{F}_{1} \cdot \ddot{\mathrm{U}}_{8}+\mathrm{F}_{2} \cdot \ddot{\mathrm{U}}_{7}+\mathrm{F}_{3} \cdot \ddot{\mathrm{U}}_{6}+\mathrm{F}_{4} \cdot \ddot{\mathrm{U}}_{5}+\mathrm{F}_{5} \cdot \ddot{\mathrm{U}}_{4}+\mathrm{F}_{6} \cdot \ddot{\mathrm{U}}_{3}+\mathrm{F}_{7} \cdot \ddot{\mathrm{U}}_{2}+\mathrm{F}_{8} \cdot \ddot{\mathrm{U}}_{1}=\mathrm{F}_{14}
\end{aligned}
$$

n elemanlı bir kümenin herhangi iki ardışık eleman içermeyen toplam alt küme sayısının $\mathrm{F}_{\mathrm{n}+2}=1+\mathrm{n}+\ddot{\mathrm{U}}_{\mathrm{n}-2}+\mathrm{F}_{1} \cdot \ddot{\mathrm{U}}_{\mathrm{n}-4}+\mathrm{F}_{2} \cdot \ddot{\mathrm{U}}_{\mathrm{n}-5}+\mathrm{F}_{3} \cdot \ddot{\mathrm{U}}_{\mathrm{n}-6}+\ldots+\mathrm{F}_{\mathrm{n}-5} \cdot \ddot{\mathrm{U}}_{2}+\mathrm{F}_{\mathrm{n}-4} \ddot{\mathrm{U}}_{1}$ olduğu gör ülmektedir.

$n$ yerine $(n-2)$ yazarak $F_{n}$ eşitliğini elde edersek:

$$
\mathrm{F}_{\mathrm{n}+2}=\mathrm{n}-1+\ddot{\mathrm{U}}_{\mathrm{n}-4}+\mathrm{F}_{1} \cdot \ddot{\mathrm{U}}_{\mathrm{n}-6}+\mathrm{F}_{2} \cdot \ddot{\mathrm{U}}_{\mathrm{n}-7}+\mathrm{F}_{3} \cdot \ddot{\mathrm{U}}_{\mathrm{n}-8}+\ldots+\mathrm{F}_{\mathrm{n}-7} \cdot \ddot{\mathrm{U}}_{2}+\mathrm{F}_{\mathrm{n}-6} \cdot \ddot{\mathrm{U}}_{1}
$$

Buradan hareketle Fibonacci Sayıları için yeni bir tanım yapabiliriz:

$$
\mathrm{F}_{\mathrm{n}}= \begin{cases}\mathrm{n}-1, & 1<\mathrm{n}<5 \\ \mathrm{n}-1+\ddot{\mathrm{U}}_{\mathrm{n}-4}, & 5 \leq \mathrm{n}<7 \\ \mathrm{n}-1+\ddot{\mathrm{U}}_{\mathrm{n}-4}+\sum_{\mathrm{k}=1}^{\mathrm{n}-6} \mathrm{~F}_{\mathrm{k}}+\ddot{\mathrm{U}}_{\mathrm{n}-\mathrm{k}-5}, & 7 \leq \mathrm{n}\end{cases}
$$

n elemanlı kümenin alt küme sayılarından $(n+2)$. Fibonacci Sayısı elde edildiğinden bu tanım ile $\mathrm{F}_{1}$ elde edilemez.

$$
\begin{aligned}
& \mathrm{F}_{2}=\mathrm{n}-1=1 \\
& \mathrm{~F}_{3}=\mathrm{n}-1=2 \\
& \mathrm{~F}_{4}=\mathrm{n}-1=3 \\
& \mathrm{~F}_{5}=\mathrm{n}-1+\ddot{\mathrm{U}}_{\mathrm{n}-4}=4+1=5 \\
& \mathrm{~F}_{6}=\mathrm{n}-1+\ddot{\mathrm{U}}_{\mathrm{n}-4}=5+3=8 \text { için tanımın doğru olduğu aşikârdır. }
\end{aligned}
$$


n $\geq 7$ için yazdı̆̆ımız tanımı ispatlamamız gerekir. İspat için gerekli olan tanımlar:

Tanım 1: Fibonacci dizisi, her sayının kendinden hemen önceki iki sayının toplanması sonucu oluşan bir sayı dizisidir. Bu durumda, genel olarak n’inci Fibonacci Sayısı $\mathrm{F}_{\mathrm{n}}$ şu şekilde ifade edilir:

$$
\begin{aligned}
& \mathrm{F}_{1}=1, \\
& \mathrm{~F}_{2}=1, \\
& \mathrm{~F}_{\mathrm{n}}=\mathrm{F}_{\mathrm{n}-1}+\mathrm{F}_{\mathrm{n}-2} \quad \mathrm{n} \geq 3
\end{aligned}
$$

Tanım 2: $\ddot{U}_{n}$ : n. Üçgensel Sayısı olmak üzere $\ddot{U}_{n}=\frac{n(n+1)}{2}$ olup; $\ddot{U}_{n}-\ddot{U}_{n-1}=n^{\prime}$ dir.

Teorem 1: $\mathrm{n} \geq 7$ ve $\mathrm{n} \in \mathrm{N}$ olmak üzere her Fibonacci Sayısı:

$\mathrm{F}_{n}=\mathrm{n}-1+\ddot{\mathrm{U}}_{\mathrm{n}-4}+\mathrm{F}_{1} \cdot \ddot{\mathrm{U}}_{\mathrm{n}-6}+\mathrm{F}_{2} \cdot \ddot{\mathrm{U}}_{\mathrm{n}-7}+\mathrm{F}_{3} \cdot \ddot{\mathrm{U}}_{\mathrm{n}-8}+\ldots+\mathrm{F}_{\mathrm{n}-\mathrm{7}} \cdot \ddot{\mathrm{U}}_{2}+\mathrm{F}_{\mathrm{n}-6} \cdot \ddot{\mathrm{U}}_{1}=\mathrm{n}-1+\ddot{\mathrm{U}}_{\mathrm{n}-4}+\sum_{\mathrm{k}=1}^{\mathrm{n}-6} \mathrm{~F}_{\mathrm{k}}+\ddot{\mathrm{U}}_{\mathrm{n}-\mathrm{k}-5}$ şeklinde ifade edilir.

İspat 1: İspatı tümevarımla aşağıdaki şekilde yaparız.

Önermemiz P(n): $F_{n}=n-1+\ddot{U}_{n-4}+F_{1} \cdot \ddot{U}_{n-6}+F_{2} \cdot \ddot{U}_{n-7}+F_{3} \cdot \ddot{U}_{n-8}+\ldots+F_{n-7} \cdot \ddot{U}_{2}+F_{n-6} \cdot \ddot{U}_{1}$ şeklindedir.

1. $\mathrm{n}=7$ için $\mathrm{F}_{7}=6+\ddot{\mathrm{U}}_{3}+\mathrm{F}_{1} \cdot \ddot{\mathrm{U}}_{1}=6+6+1.1=13$ olduğundan $\mathrm{P}(7)$ doğrudur.

2. $\mathrm{n}=8$ için $\mathrm{F}_{8}=7+\ddot{\mathrm{U}}_{4}+\mathrm{F}_{1} \cdot \ddot{\mathrm{U}}_{2}+\mathrm{F}_{2} \cdot \ddot{\mathrm{U}}_{1}=7+10+1.3+1.1=21$ olduğundan $\mathrm{P}(8)$ doğrudur.

3. $\mathrm{n}=\mathrm{k}-1$ için ve $\mathrm{n}=\mathrm{k}$ için önerme doğru olsun. Yani $\mathrm{P}(\mathrm{k}-1): \mathrm{F}_{\mathrm{k}-1}=\mathrm{k}-2+\ddot{\mathrm{U}}_{\mathrm{k}-5}+\mathrm{F}_{1} \cdot \ddot{\mathrm{U}}_{\mathrm{k}-7}+\mathrm{F}_{2}$. $\ddot{\mathrm{U}}_{\mathrm{k}-8}+\ldots+\mathrm{F}_{\mathrm{k}-8} \cdot \ddot{\mathrm{U}}_{2}+\mathrm{F}_{\mathrm{k}-7} \cdot \ddot{\mathrm{U}}_{1}$ ve P $(\mathrm{k}): \mathrm{F}_{\mathrm{k}}=\mathrm{k}-1+\ddot{\mathrm{U}}_{\mathrm{k}-4}+$

$\mathrm{F}_{1} \cdot \ddot{\mathrm{U}}_{\mathrm{k}-6}+\mathrm{F}_{2} \cdot \ddot{\mathrm{U}}_{\mathrm{k}-7}+\mathrm{F}_{3} \cdot \ddot{\mathrm{U}}_{\mathrm{k}-8}+\ldots+\mathrm{F}_{\mathrm{k}-7} \cdot \ddot{\mathrm{U}}_{2}+\mathrm{F}_{\mathrm{k}-6} \cdot \ddot{\mathrm{U}}_{1}$ olsun.

$n=k+1$ için $P(k+1): F_{k+1}=k+\ddot{U}_{k-3}+F_{1} \cdot \ddot{U}_{k-5}+F_{2} \cdot \ddot{U}_{k-6}+F_{3} \cdot \ddot{U}_{k-7}+\ldots+F_{k-6} \cdot \ddot{U}_{2}+F_{k-5} \cdot \ddot{U}_{1}$ doğru olduğunu gösterelim.

Tanım 1'den $\mathrm{F}_{\mathrm{k}-1}+\mathrm{F}_{\mathrm{k}}=\mathrm{F}_{\mathrm{k}+1}$ 'dir.

$$
\begin{aligned}
\mathrm{F}_{\mathrm{k}-1}+\mathrm{F}_{\mathrm{k}}= & 2 \cdot \mathrm{k}-3+\ddot{\mathrm{U}}_{\mathrm{k}-4}+\ddot{\mathrm{U}}_{\mathrm{k}-5}+\mathrm{F}_{1} \cdot \ddot{\mathrm{U}}_{\mathrm{k}-6}+\left(\mathrm{F}_{1}+\mathrm{F}_{2}\right) \cdot \ddot{\mathrm{U}}_{\mathrm{k}-7}+\left(\mathrm{F}_{2}+\mathrm{F}_{3}\right) \cdot \ddot{\mathrm{U}}_{\mathrm{k}-8}+\left(\mathrm{F}_{4}+\mathrm{F}_{5}\right) \cdot \ddot{\mathrm{U}}_{\mathrm{k}-9}+\ldots+\left(\mathrm{F}_{\mathrm{k}-8}+\mathrm{F}_{\mathrm{k}-7}\right) \cdot \ddot{\mathrm{U}}_{2}+ \\
& \left(\mathrm{F}_{\mathrm{k}-7}+\mathrm{F}_{\mathrm{k}-6}\right) \cdot \ddot{\mathrm{U}}_{1} \\
= & 2 \cdot \mathrm{k}-3+\ddot{\mathrm{U}}_{\mathrm{k}-4}+\ddot{\mathrm{U}}_{\mathrm{k}-5}+\mathrm{F}_{1} \cdot \ddot{\mathrm{U}}_{\mathrm{k}-6}+\mathrm{F}_{3} \cdot \ddot{\mathrm{U}}_{\mathrm{k}-7}+\mathrm{F}_{4} \cdot \ddot{\mathrm{U}}_{\mathrm{k}-8}+\mathrm{F}_{5} \cdot \ddot{\mathrm{U}}_{\mathrm{k}-9}+\ldots+\mathrm{F}_{\mathrm{k}-6} \cdot \ddot{\mathrm{U}}_{2}+\mathrm{F}_{\mathrm{k}-5} \cdot \ddot{\mathrm{U}}_{1}{ }^{\prime} \mathrm{dir} .
\end{aligned}
$$

$\mathrm{F}_{1}=\mathrm{F}_{2}=1$ olduğundan $\ddot{\mathrm{U}}_{\mathrm{k}-6}$ ve $\ddot{\mathrm{U}}_{\mathrm{k}-5}$ 'in katsayılar1 yeniden düzenlenirse;

$$
\begin{aligned}
& =2 \cdot \mathrm{k}-3+\ddot{\mathrm{U}}_{\mathrm{k}-4}+\mathrm{F}_{1} \cdot \ddot{\mathrm{U}}_{\mathrm{k}-5}+\mathrm{F}_{2} \cdot \ddot{\mathrm{U}}_{\mathrm{k}-6}+\mathrm{F}_{3} \cdot \ddot{\mathrm{U}}_{\mathrm{k}-7}+\mathrm{F}_{4} \cdot \ddot{\mathrm{U}}_{\mathrm{k}-8}+\mathrm{F}_{5} \cdot \ddot{\mathrm{U}}_{\mathrm{k}-9}+\ldots+\mathrm{F}_{\mathrm{k}-6} \cdot \ddot{\mathrm{U}}_{2}+\mathrm{F}_{\mathrm{k}-5} \cdot \ddot{\mathrm{U}}_{1} \\
& =\mathrm{k}+(\mathrm{k}-3)+\ddot{\mathrm{U}}_{\mathrm{k}-4}+\mathrm{F}_{1} \cdot \ddot{\mathrm{U}}_{\mathrm{k}-5}+\mathrm{F}_{2} \cdot \ddot{\mathrm{U}}_{\mathrm{k}-6}+\mathrm{F}_{3} \cdot \ddot{\mathrm{U}}_{\mathrm{k}-7}+\mathrm{F}_{4} \cdot \ddot{\mathrm{U}}_{\mathrm{k}-8}+\mathrm{F}_{5} \cdot \ddot{\mathrm{U}}_{\mathrm{k}-9}+\ldots+\mathrm{F}_{\mathrm{k}-6} \cdot \ddot{\mathrm{U}}_{2}+\mathrm{F}_{\mathrm{k}-5} \cdot \ddot{\mathrm{U}}_{1}
\end{aligned}
$$

Tanım 2'den $\ddot{U}_{\mathrm{k}-3}=(\mathrm{k}-3)+\ddot{\mathrm{U}}_{\mathrm{k}-4}$ olduğundan

$$
\begin{aligned}
& =\mathrm{k}+\ddot{\mathrm{U}}_{\mathrm{k}-3}+\mathrm{F}_{1} \cdot \ddot{\mathrm{U}}_{\mathrm{k}-5}+\mathrm{F}_{2} \cdot \ddot{\mathrm{U}}_{\mathrm{k}-6}+\mathrm{F}_{3} \cdot \ddot{\mathrm{U}}_{\mathrm{k}-7}+\mathrm{F}_{4} \cdot \ddot{\mathrm{U}}_{\mathrm{k}-8}+\mathrm{F}_{5} \cdot \ddot{\mathrm{U}}_{\mathrm{k}-9}+\ldots+\mathrm{F}_{\mathrm{k}-6} \cdot \ddot{\mathrm{U}}_{2}+\mathrm{F}_{\mathrm{k}-5} \cdot \ddot{\mathrm{U}}_{1} \\
& =\mathrm{F}_{\mathrm{k}+1} \text { 'dir. }
\end{aligned}
$$

Yani $P(k+1)$ de doğru olur. Böylece $P(n)$ önermesinin $n=k-1$ ve $n=k$ için doğru ise $n=k+1$ için de doğru olacağını gösterdik. Böylece $P(n)$ önermesi her $n \geq 7$ doğal sayısı için doğrudur.

\section{SONUÇ VE TARTIŞMA}

“ $\{1,2,3,4,5, \ldots, \mathrm{n}\}$ kümesinin herhangi iki ardışık tam sayı içermeyen kaç alt kümesi vardır?” probleminden yola çıkılan bu çalışmada, bir Fibonacci Sayısında gizli olan Üçgensel Sayılar keşfedilmiştir. Fibonacci Sayıları, Üçgensel Sayıların toplamı alınarak: 
$\mathrm{F}_{\mathrm{n}}= \begin{cases}\mathrm{n}-1, & 1<\mathrm{n}<5 \\ \mathrm{n}-1+\ddot{\mathrm{U}}_{\mathrm{n}-4}, & 5 \leq \mathrm{n}<7 \\ \mathrm{n}-1+\ddot{\mathrm{U}}_{\mathrm{n}-4}+\sum_{\mathrm{k}=1}^{\mathrm{n}-6} \mathrm{~F}_{\mathrm{k}}+\ddot{\mathrm{U}}_{\mathrm{n}-\mathrm{k}-5}, & 7 \leq \mathrm{n}\end{cases}$

şeklinde tanımlanmıştır. $\mathrm{n} \geq 7$ ve $\mathrm{n} \in \mathrm{N}$ olmak üzere her Fibonacci Sayısı:

$\mathrm{F}_{\mathrm{n}}=\mathrm{n}-1+\ddot{\mathrm{U}}_{\mathrm{n}-4}+\mathrm{F}_{1} \cdot \ddot{\mathrm{U}}_{\mathrm{n}-6}+\mathrm{F}_{2} \cdot \ddot{\mathrm{U}}_{\mathrm{n}-7}+\mathrm{F}_{3} \cdot \ddot{\mathrm{U}}_{\mathrm{n}-8}+\ldots+\mathrm{F}_{\mathrm{n}-7} \cdot \ddot{\mathrm{U}}_{2}+\mathrm{F}_{\mathrm{n}-6} \cdot \ddot{\mathrm{U}}_{1}=\mathrm{n}-1+\ddot{\mathrm{U}}_{\mathrm{n}-4}+\sum_{\mathrm{k}=1}^{\mathrm{n}-6} \mathrm{~F}_{\mathrm{k}}+\ddot{\mathrm{U}}_{\mathrm{n}-\mathrm{k}-5}$ şeklinde ifade edilerek ispatı Tümevarım Yöntemiyle yapılmıştır.

$\{1,2,3,4,5, \ldots, n\}$ kümesinin iki ardışık tam sayı içermeyen alt küme sayısı Fibonacci Sayısı olup kendinden önceki iki sayının toplamı ile hesaplanmıştır.

\section{KAYNAKLAR}

Aksoy, E., Sarı, Y. (2017). "Parabol Serilerinin Eklemeli Toplamlar Dizisinin Terimleri ile Üçgensel Sayılar Arasındaki İlişkiler". TÜBİTAK 48. Lise Öğrencileri Araştırma Projeleri Final Yarışması Özet Kitapçı̆̆ 1,$48 ; 124$.

Bulut, F. (2017). "Pascal Üçgeni, Kombinasyon ve Tümevarım Kullanarak Fibonacci Dizisinin N. Elemanını Bulma". El-Cezerî Fen ve Mühendislik Dergisi, 4(3); 429-435.

Koşar, E. (2013). "Fibonacci Sayılarında $\mathrm{F}_{n} \mathrm{p} \mid \mathrm{F}_{\mathrm{m}}$ Şartının Araştırılması”. TÜBİTAK 44. Ortaöğretim Öğrencileri Araştırma Projeleri Final Yarışması Özet Kitapçı̆̆ı $144 ; 127$.

Özer, A., Ünlü, K. (2009). “Fibonacci Ağac1”. TÜBİTAK 40. Ortaöğretim Öğrencileri Araştırma Projeleri Final Yarışması Özet Kitapçı̆̆ı, 40; 90.
Sertöz, S. (1997). Matematiğin Aydınlık Dünyası. TÜBİTAK Popüler Bilim Kitapları, 6. Basım. Ankara.

Takkın, B. K., Korkmaz, A. (2015). “Alt Kümelerin Üretici Fonksiyonları". TÜBİTAK 46. Ortaöğretim Öğrencileri Araştırma Projeleri Final Yarışması Özet Kitapçı $\breve{1} 1,46 ; 118$.

Taşgın, S., Taşgın. S. (2016). “Alt Küme Elemanlarının Toplamını Bulmada Pascal Üçgeni Yaklaşımı". TÜBİTAK 47. Ortaöğretim Öğrencileri Araştırma Projeleri Final Yarışması Özet Kitapçı̆̆ı, $47 ; 137$.

Uzun, M. F., Keleştemur, S. E. (2012). “Olasılık ve Fibonacci Dizisi Arasındaki İlişki". TÜBİTAK 43. Ortaöğretim Öğrencileri Araştırma Projeleri Final Yarışması Özet Kitapçı̆̆ı, 43; 126.

Wells, D. (1997). Matematiğin Gizli Dünyası, Türkçesi: Dr. Selçuk Alsan. Sarmal Yayınevi, Birinci Bask1. İstanbul. 